Trascender, Contabilidad y Gestión Núm. 11 (mayo - agosto del 2019). ISSN: 2448-6388. Universidad de Sonora. Departamento de Contabilidad. Reserva de Derechos 04-2015-04172070800-203.

\title{
Temas relevantes de aprendizaje del área de costos en los estudiantes de una universidad pública
}

Relevant cost learning topics in students at a public university

\begin{abstract}
Vanessa Carolina Hernández Monsreal ${ }^{1}$; Patricia Hernández García ${ }^{2}$; Ma. Guadalupe de Socorro Laura Dávalos Verástegui ${ }^{3}$
\end{abstract}

\section{Resumen}

En la actualidad en México, como en diversas partes del mundo, se están dando cambios sorprendentes en los ámbitos financieros, económicos, entre otros, que afectan tanto a las empresas y a los individuos. El reto de las organizaciones es encontrar aquel sistema de costos que se viable y apropiado para la empresa, enfocándolo a la reducción de costos, aumento de ingresos, así como de producción, brindándole a los directivos, mandos altos, la gerencia para la correcta toma de decisiones debidamente sustentadas, logrando competir en los mercados nacionales e internacionales. Por lo anterior el objetivo general de la presente investigación fue conocer cuáles fueron los temas más relevantes de aprendizaje del área de costos de los estudiantes de universidad pública, para ello se realizó una investigación cuantitativa con un cuestionario a 11 grupos de 5 alumnos con la intención de conocer el nivel de conocimiento que se tiene en el área de costos, lo cual dio como resultado un nivel bajo en el conocimiento de costos por parte de la muestra, sugiriendo por tanto desarrollar estrategias necesarias para mejorar el nivel de conocimiento de los alumnos a fin de mejorar el desempeño de la organización, y con ello apoyar al desarrollo de la misma con nuevas formas de trabajo.

Palabras clave: costos, universidad pública, aprendizaje.

DOI: https://doi.org/10.36791/tcg.v11i0.64

JEL: D83. Búsqueda, aprendizaje, información y conocimiento.

Recibido: 16 de diciembre de 2018.

Aceptado: 24 de junio de 2019.

\footnotetext{
${ }^{1}$ Vanessa Carolina Hernández Monsreal. Instituto Tecnológico de Chetumal. Correo: vanehernandez06@hotmail.com

${ }^{2}$ Patricia Hernández García. Universidad Autónoma de San Luis Potosí. Correo: patricia.hernandez@uaslp.mx

${ }^{3}$ Ma. Guadalupe de Socorro Laura Dávalos Verástegui. Universidad Autónoma de San Luis Potosí. Correo: mgsldv@hotmail.com
} 


\begin{abstract}
Currently in Mexico, as in many parts of the world, surprising changes are taking place in the financial, economic, among others, which affect both companies and individuals. The challenge of the organizations is to find that cost system that is viable and appropriate for the company, focusing it on reducing costs, increasing revenues, as well as production, providing managers, senior managers, management for the correct take of duly supported decisions, managing to compete in national and international markets. Therefore, the general objective of this research was to know what were the most relevant learning topics in the area of costs of public university students, for this purpose a quantitative investigation was conducted with a questionnaire to 11 groups of 5 students with the intention of knowing the level of knowledge that is had in the area of costs, which resulted in a low level in the knowledge of costs on the part of the sample, suggesting therefore to develop necessary strategies to imp rove the level of knowledge of the students to in order to improve the performance of the organization, and thereby support its development with new forms of work.
\end{abstract}

Keywords: cost, public university, learning. 


\section{Introducción}

Las empresas actualmente se enfrentan al reto y presión por reducir costos (Demeere, Stouthuysen y Roodhooft, 2009; Kaszubski y Ebben, 2004; RodríguezMaeso, 2010; Tsai y Lai, 2007), además de generar una utilidad aún mayor. Las empresas utilizan el sistema de costos como soporte, y aprovechan su información para tomar decisiones en todos los niveles de la organización (Mejía Argueta e Higuita Salazar, 2015). Taj y Berro (2006) afirman que las empresas de manufactura desperdician alrededor del $70 \%$ de sus recursos, y además se requiere sincronizarse con los requerimientos del cliente para cubrir el flujo de materiales, desde el proveedor, a fin de reducir los costos de inventario y mejorar el nivel del servicio al cliente.

La globalización ha tenido efectos trascendentales que han generado cambios en las actividades de una empresa frente a su mercado; los consumidores cuentan con mayores opciones de bienes y servicios, que los vuelve más exigentes en la selección de sus satisfactores, debido a que buscan el mejor producto con la mejor calidad, un precio atractivo y servicio de adquisición (Guiltinan y Madden, 2007). Las empresas son grandes porque proporcionan precios competitivos y productos $\mathrm{y}$ servicio de calidad que son requeridos por los mercados a los que abastecen. Sin estos factores, ni el más grande sistema de calidad de gestión en el mundo hará que una empresa sea una empresa de éxito (Hicks, 1998).

El proceso para obtener los costos en el período de la Revolución Industrial (Cuevas, Chávez, Castillo, Caicedo y Solarte, 2004; Nakagawa, 2002) se realizó considerando los costos directos como la fuerza laboral y materias primas empleadas en el proceso de producción, además de considerar los costos indirectos. Según Mejía Argueta e Higuita Salazar (2015) los sistemas de costos deben tener la capacidad de reconocer las variaciones de costos causados por diferentes actividades con base en los objetos de estudio (cliente o producto), debido al escaso grado de sistematización de la mayor proporción de empresas que son las micro, pequeñas y medianas empresas (mipyme), así como por el costo que este proceso implicaría.

Emblemsvag (2007) define el término de costos como la medida del consumo de los recursos empleados para la demanda de las actividades y trabajo a realizar, considerando para ellos elementos como materia prima directa, talento humano directo y los costos indirectos de fabricación o servicio (García Colín, 2008).

Los métodos de costeo tradicionales enfatizan en los costos y gastos de producción, pero no existe claridad en la asignación de los costos relacionados con actividades hacia el cliente final, tales como actividades logísticas, comerciales y de mercadeo relacionadas con la entrega del producto. Algunas de estas actividades (sobre todo las indirectas) son asignadas como gastos sin tener en cuenta la naturaleza variable que está dada por el tipo de clientes, marcas y/o productos, así como por la manera en que estos gastos afectan la rentabilidad de la compañía, del canal o del cliente final directamente (Guerreiro, Rodrigues y Vazquez, 2008).

El problema identificado es el bajo nivel de conocimientos que tienen los alumnos en el área de costos, y que ello les llevará a tener menor capacidad de solución de problemas y toma de decisiones en las actividades relacionadas con este tópico. Por lo anterior el presente documento de investigación tiene como objetivo general conocer cuáles son los temas más relevantes de aprendizaje del área de costos de los estudiantes de universidad pública, con el fin de saber el conocimiento que los alumnos poseen y que les permitirán aplicar en su ejercicio laboral. La hipótesis no hay deferencias en cuanto a conocimiento entre los temas de costos. Para lo anterior se realizó en primer ligar revisión de la literatura para identificar los temas
Vanessa Carolina Hernández Monsreal; Patricia Hernández García; Ma. Guadalupe de Socorro Laura Dávalos Verástegui 
del área de costos que debieron conocer los alumnos y posteriormente desarrollar una serie de preguntas a manera de cuestionario para identificar si se conoce o no el tema, posteriormente se aplicó el cuestionario para conocer el nivel de conocimientos que se tiene de costos, concluyendo que hay diferencia significativa con los temas de costos.

\section{La revisión de la literatura en los costos}

Existen criterios enmarcados dentro de la contabilidad de gestión y la administración de costos, los cuales identifican las actividades innecesarias, a fin de disminuir costos producidos por las ineficiencias, e incrementar a la vez la calidad, tanto en las actividades de producción como en las de comercialización. Estas técnicas son adoptadas por empresas que desean liderazgo en costos para competir (Ramírez Padilla, 2008).

Para Morillo (2001) todo sistema de costos debe permitir planificar, medir y controlar los costos a cualquier nivel en la empresa, sólo así se pueden tomar decisiones coherentes que permitan maniobrar las variables (costos, ventas, activos, y otros conceptos) para incrementar la rentabilidad financiera, puesto que gran parte del comportamiento de dichas variables dependen del mejoramiento continuo, que permite a su vez el uso racional de recursos (reducción de costos y activos) y el perfeccionamiento de las actividades que añaden valor y diferenciación al producto (incremento de las unidades vendidas y precios).

De acuerdo a García Colín (2008) costo es el valor en términos monetarios de los recursos que se entregan a cambio de un bien o servicio adquirido, el cual origina beneficios presentes o futuros. Otra característica de los costos, para Amat y Soldevila (1998), es que siempre deben ser asignados a algún objeto de costos, es decir a un producto, a una fase en la elaboración del mismo o a un centro de costos. Pérez de León (1999), resalta la diferencia existente entre los términos costo y pago, indicando que este último es un desembolso de efectivo y que un costo puede implicar el diferir su pago (en magnitud y en tiempo). Es importante diferenciar entre costo y gasto; gasto es una medida de consumo relacionada con la capacidad proporcionada para realizar el trabajo, la actividad y/o el proceso (Emblemsvag, 2007).

El costo está integrado por materia prima, mano de obra directa y gastos indirectos de fabricación. Materia prima son los costos de los insumos que integran el producto más todos los gastos relacionados para que pueda ser utilizado, por ejemplo flete, impuestos aduanales, seguros, acarreos, etc.; mano de obra es el costo pagado a las personas que transforman el producto o que prestan el servicio y los costos indirectos de fabricación son aquellos que intervienen en la transformación de los productos o servicios de manera indirecta, esto es, que no se pueden identificar directamente en el producto o servicio (Ramírez Padilla, 2008).

El requerimiento indispensable de registrar la información de cada transacción comercial, detallando los gastos y costos, permite evitar la redundancia de la información y evitar ineficiencias de ello.

\section{Importancia del conocimiento de costos}

Contar con una estrategia de gajo costo permite que el precio sea el principal medio de competencia de las organizaciones, como base para una estrategia eficiente de la gestión de cotos. Según Mejía Argueta e Higuita Salazar (2015), dada la influencia del sistema de costos en el desempeño funcional de la organización, es importante que el sistema se encuentre estructurado de manera flexible para representar y relacionar costos, dependiendo de las necesidades de medición de la empresa, del sector industrial y de la cadena de suministro bajo análisis. 
Por lo tanto, se debe entender que el sistema de costos debe generar reportes financieros útiles para entidades externas vinculadas a las compañías, como inversionistas, acreedores, entidades reguladoras $\mathrm{y}$ fiscalizadoras, entre otras; además de generar estimadores financieros desagregados de costos y/o gastos directos e indirectos internos, con el fin de lograr mayor eficiencia organizacional a nivel estratégico, táctico y operativo (Cooper y Kaplan, 1998).

Los métodos de costeo se clasifican por su aplicación en tradicionales y contemporáneos. Es por ello, que en la actualidad las actividades empresariales se han ido perfeccionando con el incremento de nuevas herramientas que permiten avanzar en entornos relativamente inestables, con elevada presión competitiva, permitiendo la inserción en mercados globalizados y alcanzar los niveles de calidad y rentabilidad deseados, siendo cada día más eficientes y competitivos. Por esta razón, el aumento de las ganancias de las empresas como resultado de un elevado nivel de calidad de los servicios y una disminución de los costos, debe ser un objetivo a alcanzar (Ramírez Padilla, 2008):

- Dentro de los costos contemporáneos está el costeo por objetivo es una técnica adoptada por las empresas japonesas, para penetrar en los mercados extranjeros, el cual consiste en ofrecer un producto de calidad (satisfacción de las necesidades del cliente) y además ofrecer un precio que le asegure la demanda.

- El sistema de teoría de valor fundamenta en la percepción subjetiva y motivacional de los consumidores al comprar cierto artículo, de tal forma que el valor se incrementa al satisfacer las necesidades mejor que la competencia, a un precio que no rebase las disponibilidades del consumidor. el sistema de costo total de calidad es una cultura incorporada en la administración de las organizaciones, la cual tiene por finalidad alcanzar la excelencia en todas las dimensiones.

- El sistema justo a tiempo cuyo procedimiento ayuda a gestionar la reducción de los tiempos totales del proceso para fabricar y vender un producto (Morillo, 2001). El sistema de costo de vida del producto el cual es una metodología que trata de influenciar los costos de los productos elaborados, a partir de un diseño adecuado de tal manera que se puedan tomar acciones durante las primeras etapas del ciclo de vida (concepción, diseño y pruebas) que permitan abatir costos en las etapas de producción (Hansen y Mowen, 1996).

- El costo de servicio definido el costo de la integración de flujos de información, físicos y monetarios hasta entregar el producto al cliente final y tiene como objetivo calcular la rentabilidad de productos, clientes y canales de distribución, así como sentar las bases para el diseño de la oferta de valor para una segmentación de clientes soportada en su margen de contribución y las necesidades particulares de los clientes; esta idea se empezó a desarrollar con la gestión basada en actividades (Accenture, 2012; Freeman, Haasz, Lizzola y Seiersen, 2000; Guerreiro et al., 2008; Byrnes, 2010).

- Los sistemas de costos de calidad son los que cuantifican financieramente los costos de calidad de la organización agrupados en costos de cumplimiento y de no cumplimiento, para facilitar a la gerencia la selección de niveles de calidad que minimicen los costos de la misma. El sistema de costo total se origina de todas las actividades que previamente sean dividido y asignado, dando como resultado el costo de servir (Marteau y Perego, 2001). 
- El sistema de costeo en actividad también es conocido como el costo basado en Actividades o Costo basado en actividades (ABC) que ha tenido mayor adopción en las organizaciones se considera los factores: absorción, variable y actividad (Nara, 2011), además el método ABC se determina de abajo hacia arriba (bottom-up), ya que se inicia con actividades de menor importancia y sigue sumándolas hasta tener finalizado el análisis de toda la organización con su respectivo costeo (Mejía Argueta e Higuita Salazar, 2015), además ABC se considera la base para la correcta toma de decisiones y proporciona el beneficio de aumentar los ingresos de la organización por un tiempo más amplio (Bjornenak y Mitchell, 1999; Cagwin y Bouwman, 2002).

Las estrategias adoptadas dependen completamente de la exactitud de la estructura de costos de la empresa en su totalidad (Mejía Argueta e Higuita Salazar, 2015).

\section{Metodología}

La metodología utilizada en la presente investigación es mixta ya que se tomó información tanto cuantitativa como cualitativa para lograr el objetivo planteado. Los métodos mixtos representan un conjunto de procesos sistemáticos, empíricos y críticos de investigación e implican la recolección y el análisis de datos cuantitativos y cualitativos, así como su integración y discusión conjunta, para realizar inferencias producto de toda la información recabada y lograr un mayor entendimiento del fenómeno bajo estudio (HernándezSampieri y Mendoza, 2008). Producir datos más ricos y variados se consideraron diversas fuentes y tipos de datos, contextos o ambientes y análisis.

Como parte del procedimiento, se revisaron fuentes secundarias como artículos y libros provenientes de buscadores académicos y de bases de datos de revistas científicas como: ScienceDirect, ISI Web of Knowledge,
Scopus, Scielo, Journal Citation Reports, JStor y Google Scholar con la utilización de palabras clave relacionadas con costo de servir, costeo basado en actividades, gestión de costos, mercados emergentes y canal tradicional.

Por otro lado se realizó un cuestionario de conocimiento por expertos para ser contestado por los participantes de la muestra, el cuestionario consta de 97 preguntas de opción múltiple lo cual permitió conocer los temas que se conocen en mayor medida.

La validez del cuestionario se hizo por medio de expertos en la materia de costos, tanto para la elaboración, como para la revisión y la liberación final del instrumento. La muestra para la aplicación del cuestionario se realizó con 11 grupos de 5 personas cada uno, a fin de tener información completa para la elección de la respuesta correcta, los grupos se formaron por alumnos de la carrera de Contador > Público, Licenciado en Administración y Licenciado en Mercadotecnia, con el requisito mínimo de haber cursado una materia de costos, por lo cual fueron alumnos de quinto semestre en adelante, tanto hombres como mujeres. La intención de integrarlos de esta manera es que al momento de realizar la práctica laboral no se tiene necesariamente que trabajar de manera individual, sino que algunas actividades se realizan de manera conjunta, es decir, como un trabajo multidisciplinario, y no se pretende hacer diferencia en el análisis por género o edad, sino únicamente conocer el nivel de apropiación del conocimiento en el área de costos, dada la importancia de la misma para la organización, por el efecto que tiene en la rentabilidad de la organización. La recolección de datos se realizó en el mes de octubre de 2019 en un solo momento para todos los integrantes de la muestra, a fin de evitar que se conociera previamente la información y se generara ventaja con ello para el resto de los participantes. 
El instrumento cuenta con cuatro variables:

1) Generalidades de los costos, la cual se refiere a los conceptos de la contabilidad financiera y la contabilidad administrativa, la contabilidad de costos y la contabilidad estratégica: conceptos y diferencias; formas de organización y clasificación de las empresas; concepto, usos y clasificación de costos; sistemas de costos: tradicionales y contemporáneos.

2) Integración del costo de producción, acumulación y reporte integra los temas de Estados financieros e informes internos.

3) Elementos del costo de producción, registro y reporte considera el concepto, clasificación, descripción, registro y ejemplos de materia prima, mano de obra, gastos indirectos de fabricación

4) Sistema de costos de productos y servicios por órdenes de trabajo y por procesos considerando los temas de conceptos básicos de los sistemas de costeo; costeo por órdenes de trabajo; costeo por procesos; sistema de producción conjunta, coproductos y subproductos

En la figura 1 se puede observar que en el tema de generalidades, el cual incluye los conceptos de introducción a la contabilidad de costos, y todos los conceptos que forman la base de los costos son los que presentan mayores reactivos, tanto teóricos como prácticos y de aplicación en la organización, lo cual muestra como son las bases de la información en la contabilidad de costos y lo que se espera que los alumnos aprendan para que puedan hacer aplicación inmediata de los conceptos, o bien para que puedan contar con información generar de la misma. Las ventajas generadas con esta estructura es que se puede asegurar que las bases de los costos son las adecuadas, y les permitirá incrementar su conocimiento posterior.

Figura 1. Integración del instrumento de medición

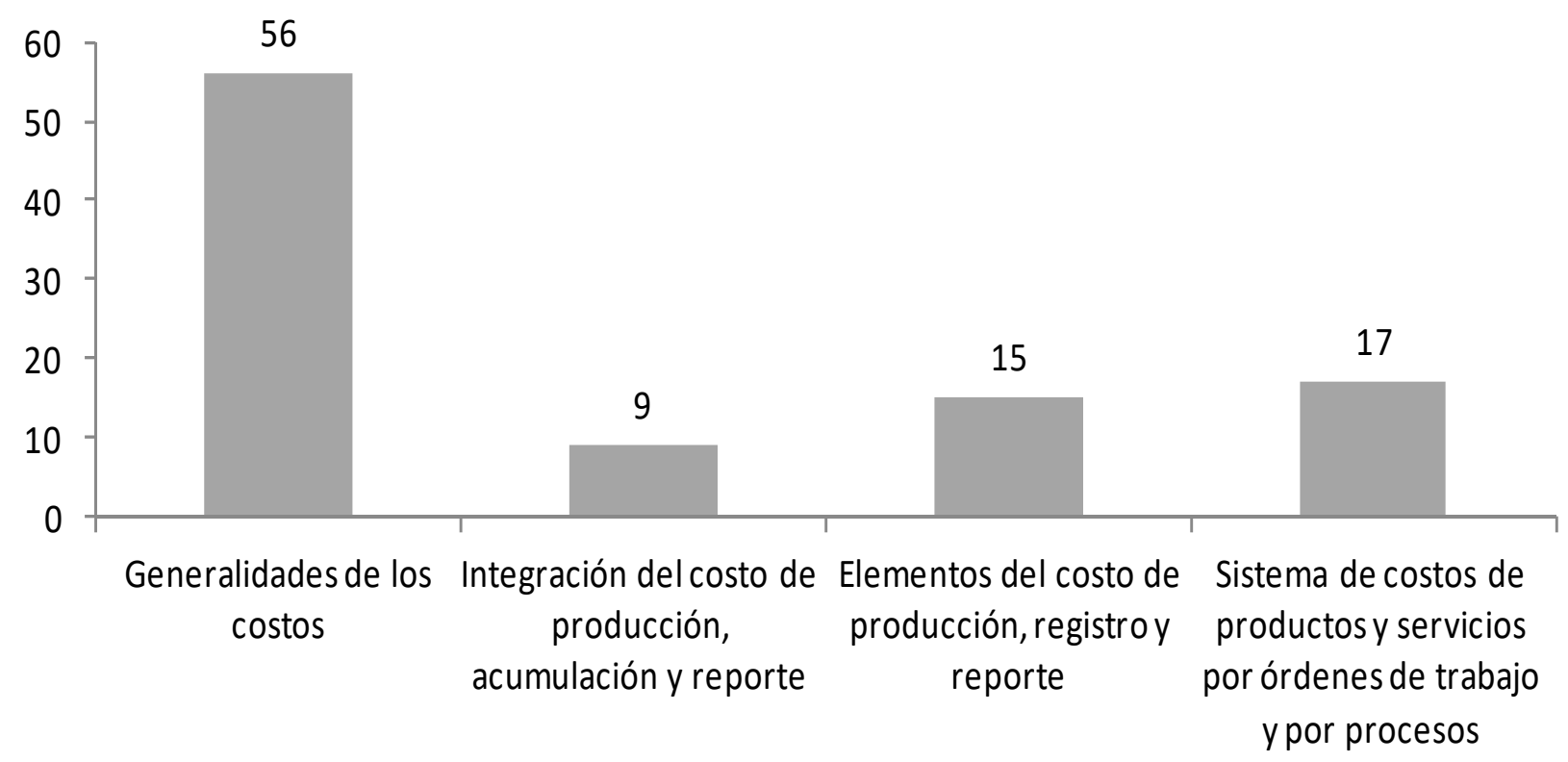

Fuente: Elaboración propia. 


\section{Resultados}

Con la intención de cumplir el objetivo de investigación que indica conocer cuáles son los temas más relevantes de aprendizaje del área de costos de los estudiantes de universidad pública, se realizó un instrumento el cual está integrado por cuatro variables como generalidades de los costos; integración del costo de producción, acumulación y reporte, elementos del costo de producción, registro y reporte y sistema de costos de productos y servicios por órdenes de trabajo y por procesos. En la figura 2 se presentan los resultados con mayor acierto, el cual muestra que el tema de integración de los costos de producción es el de mayor respuestas correctas con un $85 \%$, seguido de generalidades de los costos con un $84 \%$ y en último lugar el tema de sistema de costos de productos y servicios por órdenes de trabajo y por procesos con un $56 \%$ de preguntas correctas convirtiéndose en el tema de menor conocimiento por parte de los encuestados, lo cual muestra que se tiene menor conocimiento con respecto a conceptos básicos de los sistemas de costeo, el costeo por órdenes de trabajo, el costeo por procesos, el sistema de producción conjunta y los co-productos y subproductos, haciendo con ello aún y cuando se tenga conocimiento de las generalidades de los costos, no se logra tener una aplicación inmediata de los conceptos y aplicarlos a la vida laboral.

Figura 2. Temas con mayores aciertos

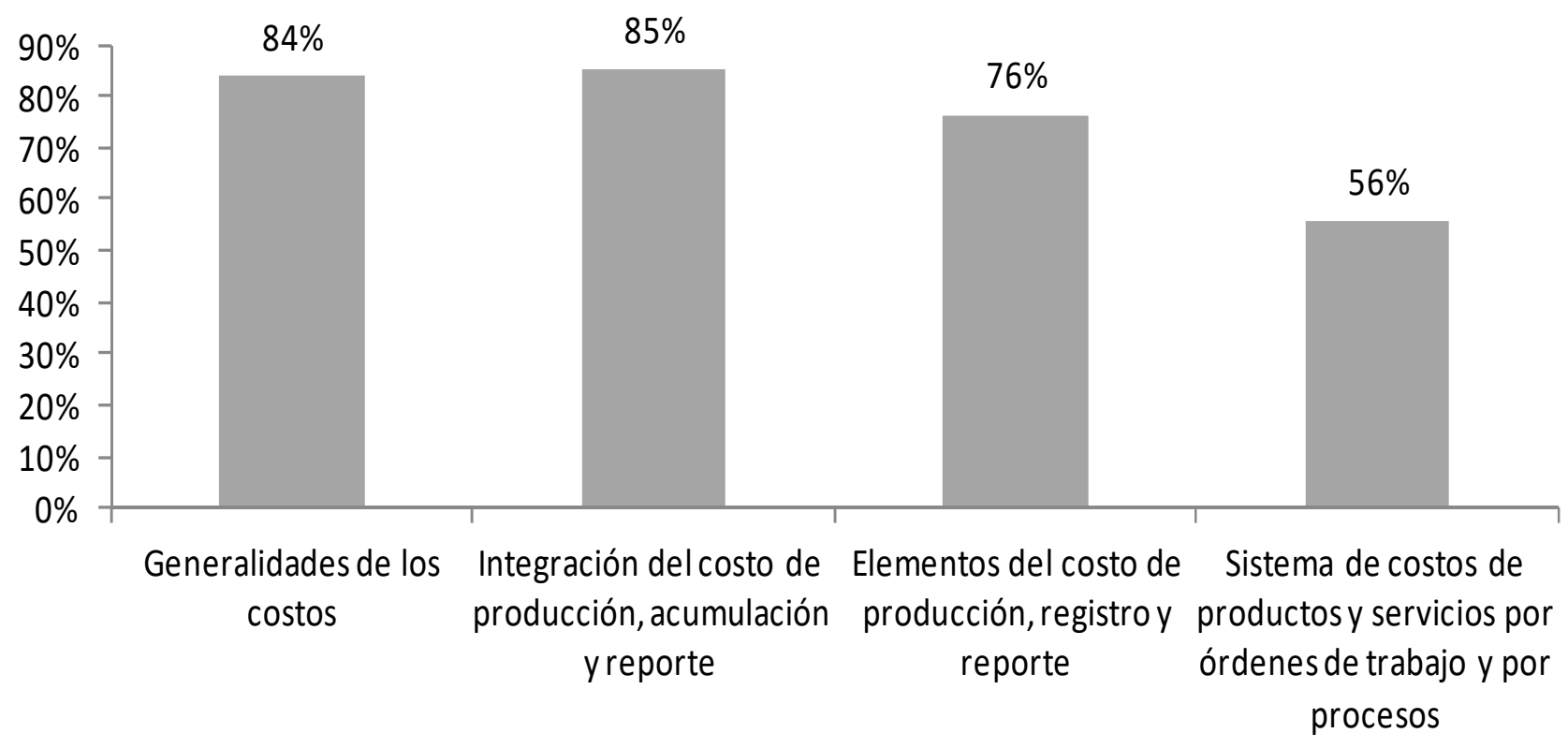

Fuente: Elaboración propia. 
En la figura 3 se muestran los resultados con menor aprobación, cabe señalar que del $100 \%$ de los reactivos no contestados (o con cero respuesta), el $80 \%$ de los mismos pertenece a las generalidades de los costos, y el $20 \%$ a los elementos del costo de producción, registro y reporte. Con lo anterior se puede observar por tanto que el mayor número de reactivos no contestados se observan en las bases de los costos, es decir, en el tema de las generalidades de los costos, lo cual pone en consideración la necesidad de establecer estrategias necesarias para mejorar los resultados, al considerar este tema como base para mejorar el desempeño en el área. Cabe mencionar también que los reactivos de integración del costo de producción, acumulación y reporte, y los de sistema de costos de productos y servicios por órdenes de trabajo y por proceso fueron respondidos en su totalidad, por lo cual muestran $0 \%$ en el nivel de respuesta menor.

Figura 3. Reactivos con menor respuesta

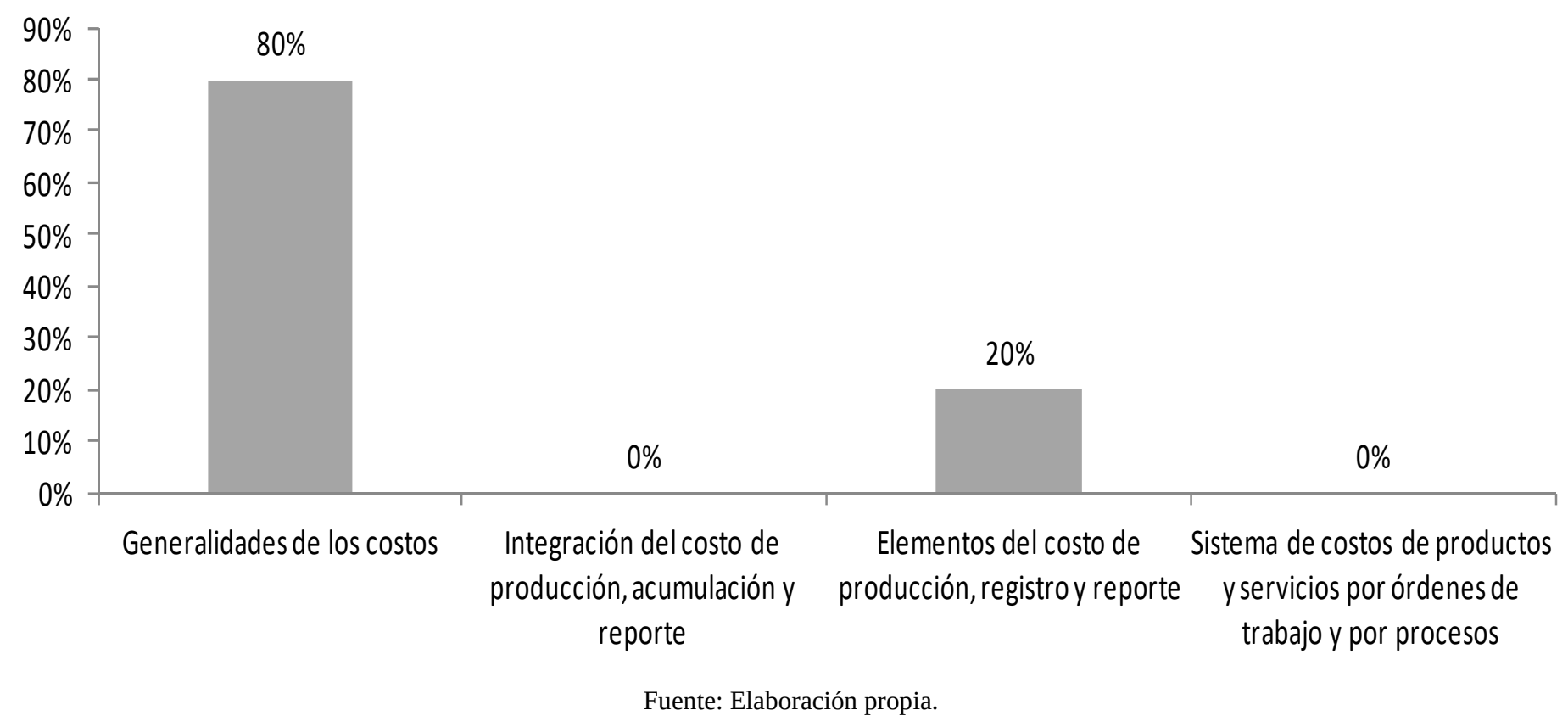

En la figura 4 se pueden conocer los resultados por cada equipo, en el cual se puede apreciar que el rango va de $56 \%$ a $77 \%$ en la totalidad de las variables encuestadas. Lo anterior tomando como base un rango de $0 \%$ a $100 \%$, por lo tanto un rango de respuestas incorrectas va de un $44 \%$ a un $23 \%$, considerando lo anterior que el total de las variables son importantes para crear una base correcta de costos y de asignación de los mismos, es relevante considerar que se tiene un alto nivel de respuestas en una zona de rechazo, pues lo correcto debe ser considerar hasta un $5 \%$ en la zona de rechazo, es decir, de respuestas incorrectas. 
Figura 4. Resultados totales de acuerdo a las respuestas del instrumento

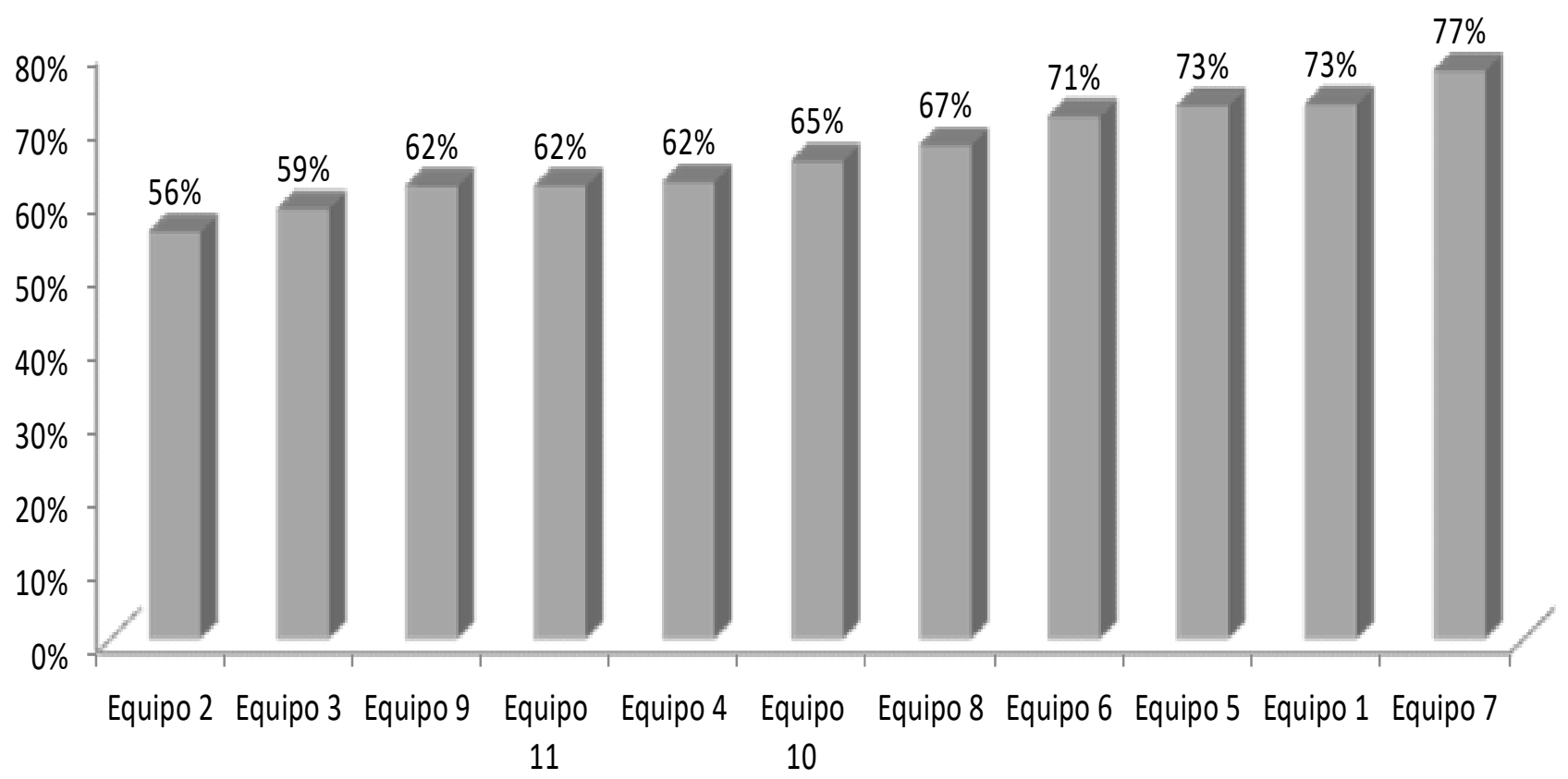

Fuente: Elaboración propia.

En la figura 5 se puede observar los resultados de la variable generalidades de los costos, el cual se aprecia que va de un rango de $59 \%$ a un $82 \%$, considerándose un nivel muy bajo para lo que debería ser un rango aceptable que es de un $95 \%$ de respuestas correctas con un de $5 \%$ de zona de rechazo. Por lo anterior es importante identificar que en promedio se conoce del tema sólo un $68 \%$, por lo que se desconoce del tema el $32 \%$, ambos casos se consideran muy bajos, dado que es la información que se conoce en esta variable pone las bases del conocimiento de la contabilidad de costos, lo cual es relevante para la construcción del conocimiento más complejo que permitirá un respaldo más relevante para la toma de decisiones. 
Figura 5. Resultados de la variable generalidades de los costos.

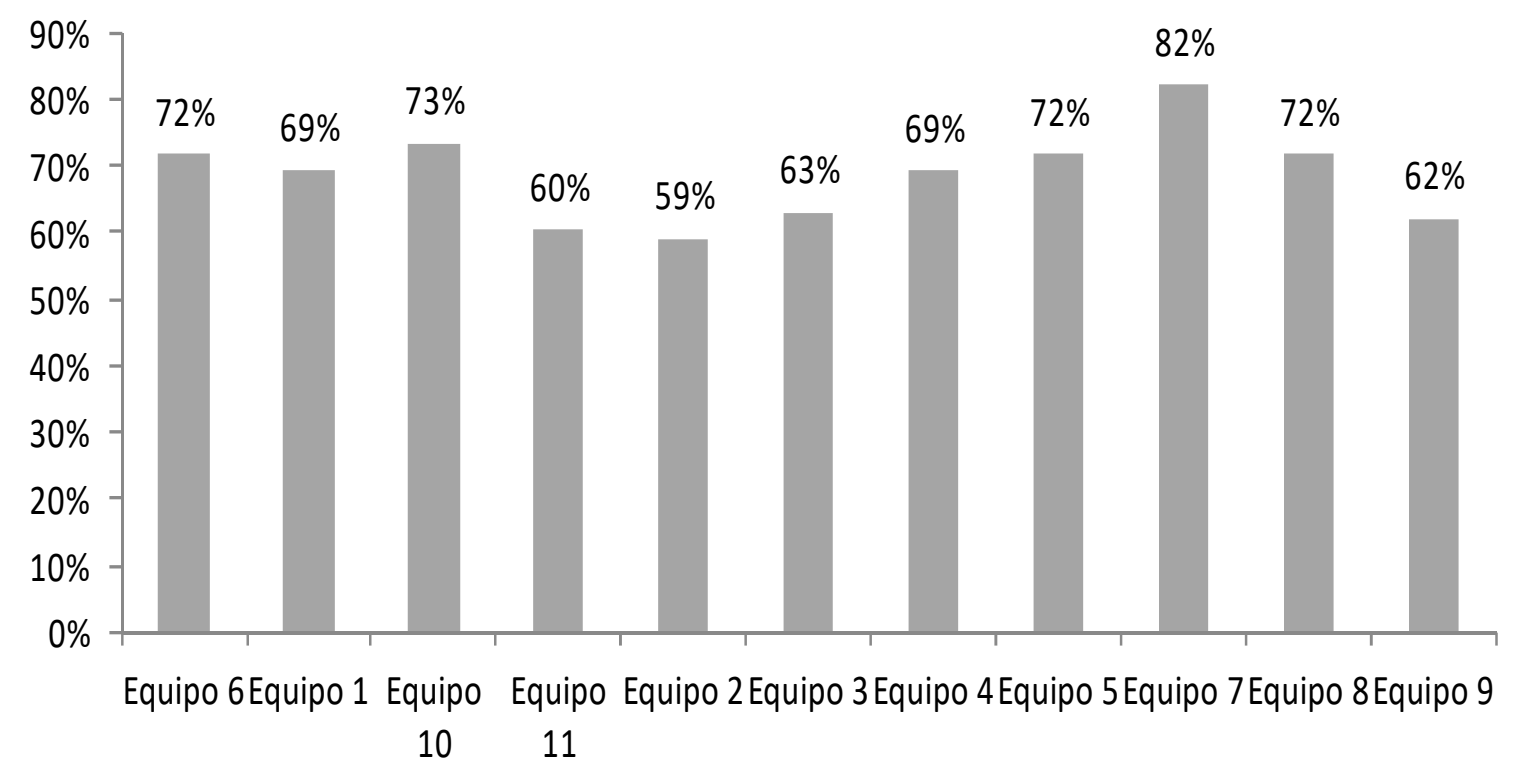

Fuente: Elaboración propia.

La figura 6 muestra los resultados generados por la pregunta de integración del costo de producción, acumulación y reporte, los cuales van de 52\% a 94\%, considerando que en promedio el valor de preguntas contestadas de manera correcta es de un $70 \%$, lo que hace que el número de preguntas contestadas de manera incorrecta es del 30\%, lo cual hace relevante que se mejore el resultado dado que la generación, acumulación y reporte de los estados financieros y la elaboración de informes internos, considerando que el nivel promedio de preguntas con respuesta correcta tiene un nivel por debajo de rango de aceptación que es el $95 \%$, por lo tanto el resultado es bajo. 
Figura 6. Resultados de la variable integración del costo de producción, acumulación y reporte

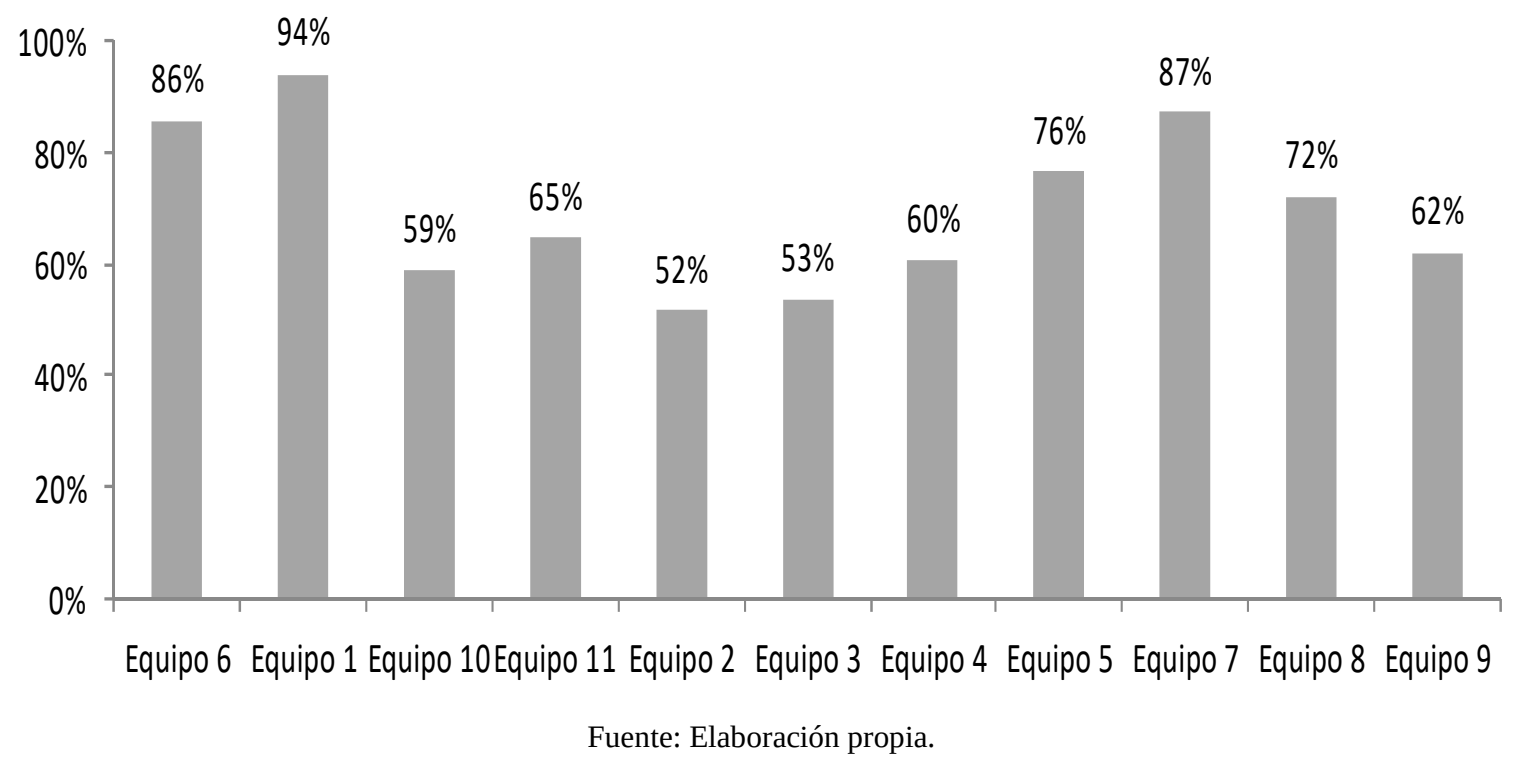

En la figura 7 se observa que la variable de elementos del costo de producción, registro y reporte presenta un rango de $50 \%$ a $73 \%$, por lo cual muestra un promedio general de $62 \%$ de preguntas contestadas de manera correcta en el tema relacionado con la clasificación, descripción, registro y ejemplos de los elementos del costo como materia prima, mano de obra y gastos indirectos de fabricación. Mostrando por otro lado que el 38\% de preguntas no contestadas en este tema es un porcentaje elevado sado que para una correcta presentación de los costos finales de producto o servicio de la organización se depende de su correcta clasificación y registro, de lo contrario se puede tener desventajas para la toma de decisiones sobre una base real, por lo anterior es relevante conocer completamente este tema, pues es la base para la toma de decisiones. 
Figura 7. Elementos del costo de producción, registro y reporte

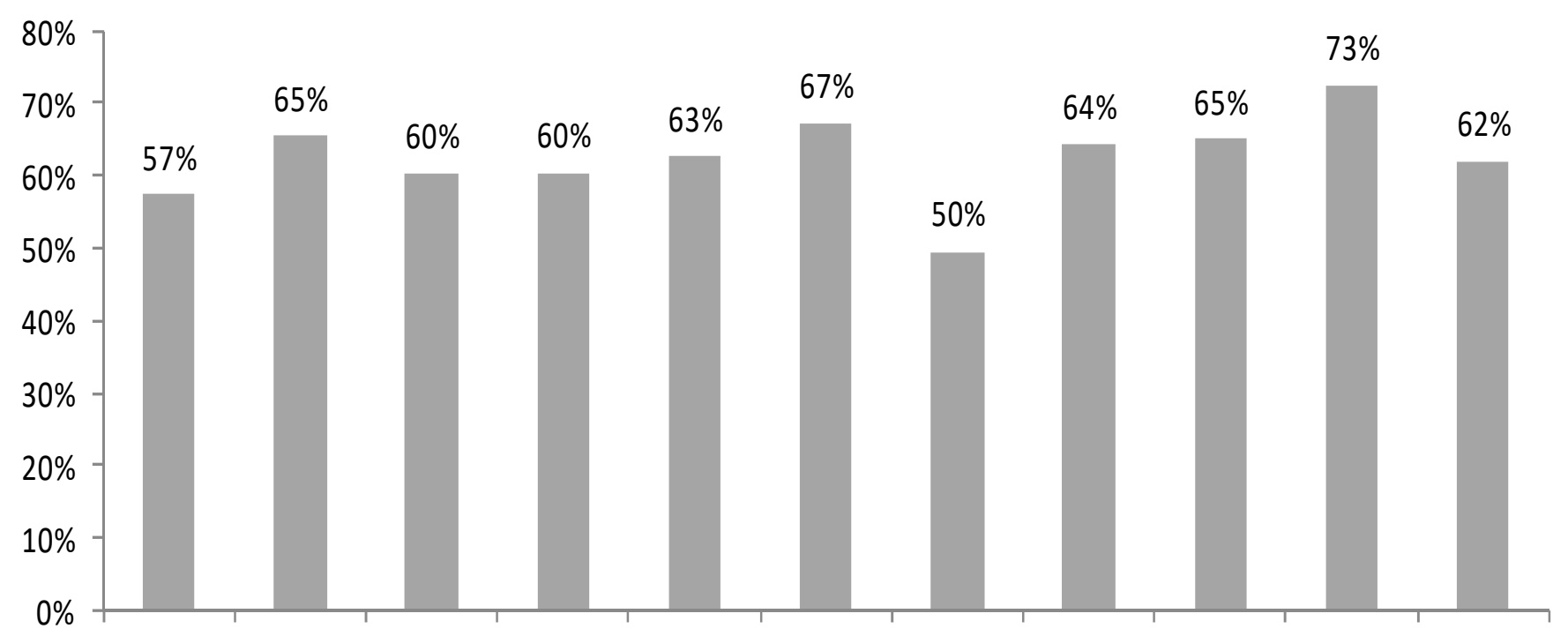

Equipo 6 Equipo 1 Equipo 10 Equipo 11 Equipo 2 Equipo 3 Equipo 4 Equipo 5 Equipo 7 Equipo 8 Equipo 9

Fuente: Elaboración propia.

En el tema de sistema de costos de productos y servicios por órdenes de trabajo y por proceso presentado en la figura 8 , muestra que los resultados están en un rango de $39 \%$ a $81 \%$ como respuesta correcta sobre los temas de sistemas de costeo por órdenes de trabajo o por procesos, así como la asignación de producción conjunta, co-productos y subproductos. Sin embargo, los resultados también demuestran que se tiene un $59 \%$ en promedio de respuestas correctas por lo cual se tiene un valor bajo sobre la base de un 95\% de respuestas correctas. Generando además que se tenga un $40.73 \%$ de respuestas no contestadas de manera incorrecta, mostrando por lo tanto una seria desventaja para el conocimiento, registro, presentación y bases para un sistema de costos adecuado al producto o servicio, afectando directamente la toma de decisiones. 
Figura 8. Sistema de costos de productos y servicios por órdenes de trabajo y por procesos

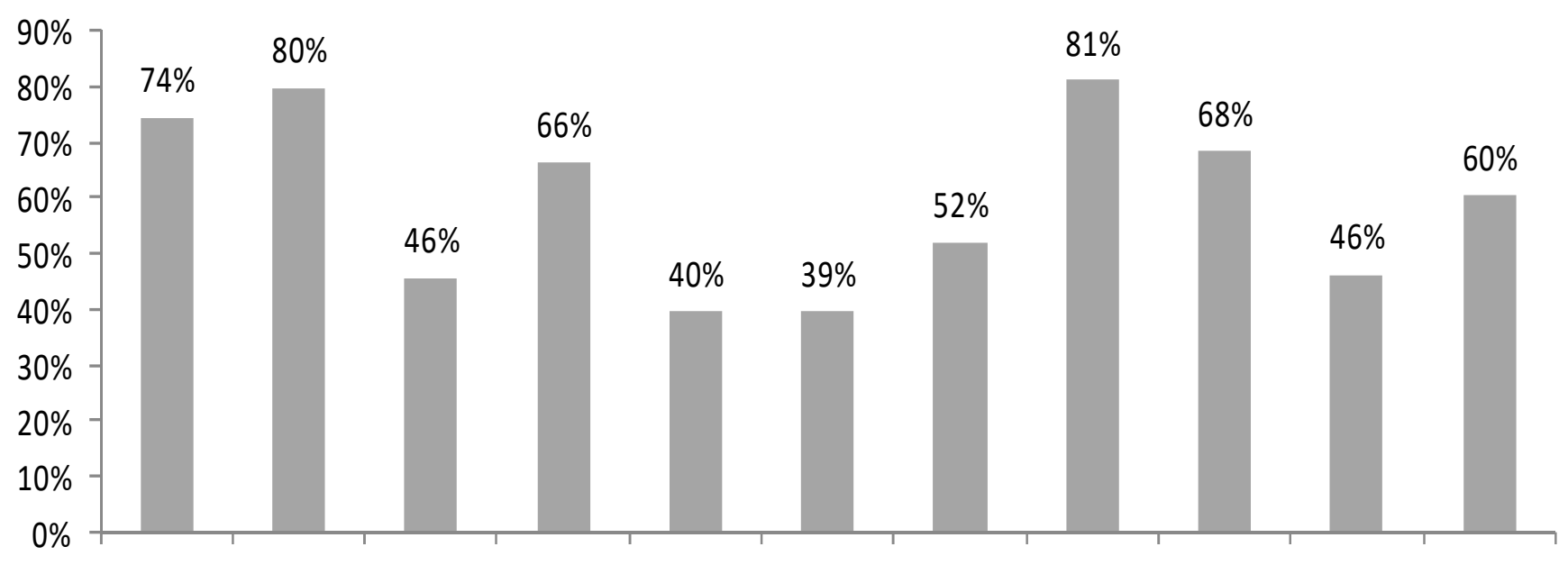

Equipo 6 Equipo 1 Equipo 10 Equipo 11 Equipo 2 Equipo 3 Equipo 4 Equipo 5 Equipo 7 Equipo 8 Equipo 9

Fuente: Elaboración propia.

En la figura 9 se aprecian los valores promedio de los resultados, por lo cual se puede apreciar que el rango promedio va de $59 \%$ a $70 \%$, lo cual es un valor bajo comparado con un rango aceptable de 95\%. Lo anterior muestra que en general no se tiene un adecuado nivel de conocimiento, siendo el menor el tema de sistema de costos y el mayor la integración del costo de producción. Por lo tanto se debe considerar los valores finales obtenidos para establecer los planes necesarios que permitan elevar el valor promedio.

Figura 9. Valor promedio de los temas de costos

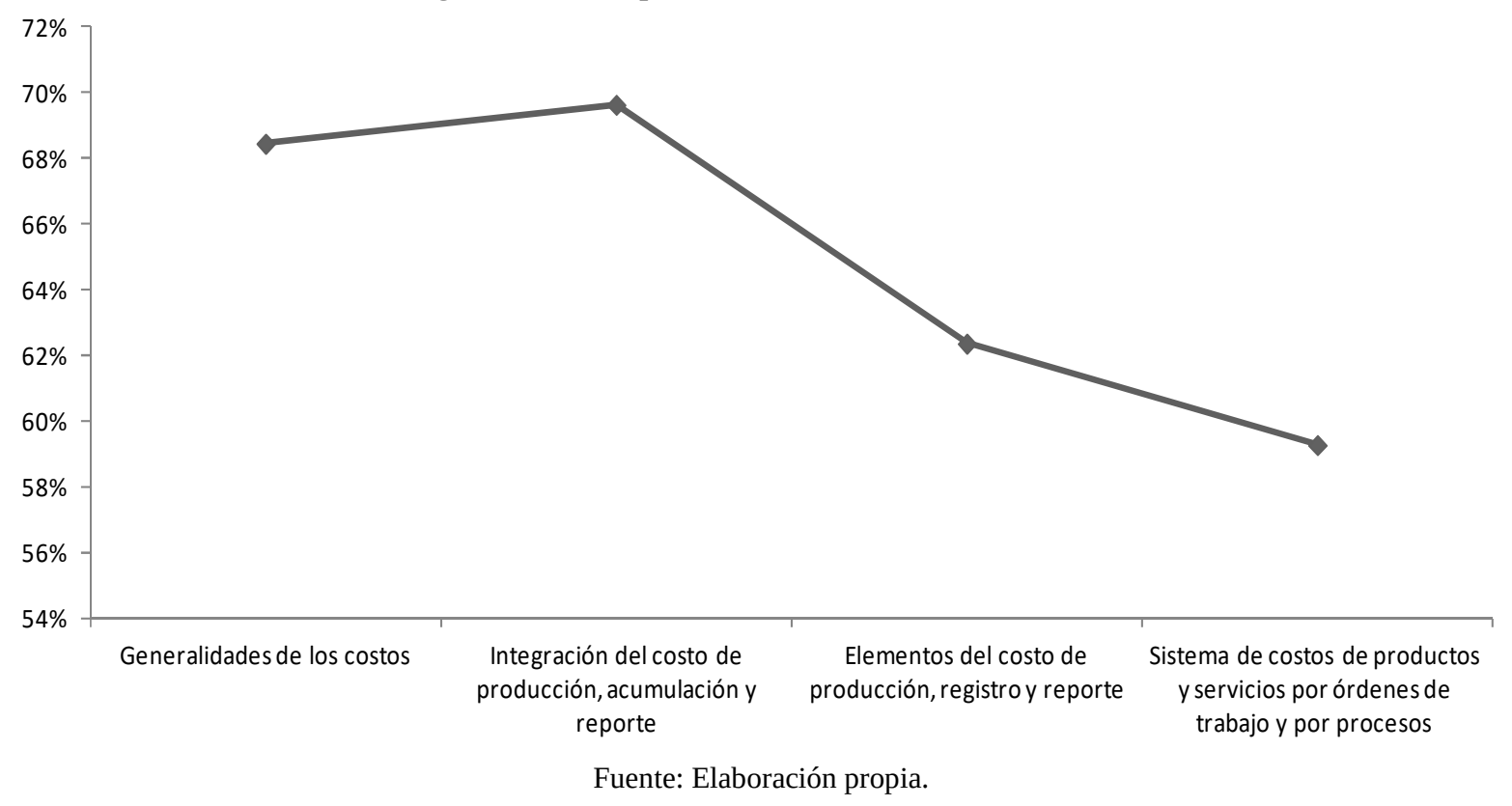


Para confirmar la hipótesis referente que no hay deferencias en cuanto a conocimiento entre los temas de costos por parte de los alumnos participantes, lo cual se muestra en la tabla 1 , en ella se observa que el valor de $\mathrm{p}>0.05$, por lo que se rechaza la hipótesis, por lo tanto se acepta de que estadísticamente hay diferencia entre los diferentes temas de costos.

Tabla 1. Análisis de varianza

\begin{tabular}{lrrrrrr}
\hline $\begin{array}{l}\text { Origen de las } \\
\text { variaciones }\end{array}$ & $\begin{array}{c}\text { Suma de } \\
\text { cuadrados }\end{array}$ & $\begin{array}{c}\text { Grados de } \\
\text { libertad }\end{array}$ & $\begin{array}{c}\text { Promedio de los } \\
\text { cuadrados }\end{array}$ & F & Probabilidad & F \\
\hline Entre grupos & 0.0723 & 3 & 0.0241 & 1.8259 & 0.1598 & 2.8662 \\
$\begin{array}{l}\text { Dentro de los } \\
\text { grupos }\end{array}$ & 0.47514 & 36 & 0.0131 & & \\
Total & 0.54744 & 39 & & & & \\
\hline
\end{tabular}

Fuente: Elaboración propia.

\section{Conclusiones}

Las organizaciones necesitan información relevante en el tema de costos, ya que esto les permitirá establecer los precios correctos, la gestión eficiente de los recursos y la mejora a corto y largo plazo de los resultados de la organización. Conocer sobre los temas de costos es básico para cualquier administrador o encargado de la gestión de recursos, por lo cual los estudiantes de la licenciatura en Contaduría Pública y licenciatura en Administración, como futuros encargados de dichas áreas, deberán conocer estos temas a profundidad. Una forma de conocer si los estudiantes cuentan con la preparación necesaria, es aplicando una serie de exámenes que les permitan medir el nivel de conocimiento obtenido, otra opción adicional es presentar un examen general de certificación o finalmente dar seguimiento a los puestos en los cuales se contratan los alumnos o egresados y con ello conocer cuántos se contratan con la responsabilidad de costos.
Por la importancia del tema, se inicia la presente investigación, la cual tiene por objetivo general el conocer cuáles son los temas más relevantes de aprendizaje del área de costos de los estudiantes de una universidad pública, con el fin de saber el conocimiento que los alumnos poseen y que les permitirán aplicar en su ejercicio laboral. Para ello se aplicó una serie de preguntar a 11 equipos formados por cinco personas del quinto semestre en adelante. Cada equipo fue contestando las preguntas y fueron evaluándose, considerando si la respuesta era o no correcta y el tiempo que tardaban en responder cada pregunta.

De acuerdo a los resultados el tema de integración del costo de producción, acumulación y reporte es el que tiene mayor nivel de respuesta, con un promedio de $70 \%$ de respuestas correctas, con ello se muestra que el tema de generación de información financiera, principalmente la generación de los informes tanto para efectos internos como para efectos externos, es decir, como base para la 
toma de decisiones relacionadas con la gestión adecuada de los recursos, como para cumplir con requisitos legales, financieros $\mathrm{y}$ comerciales entre otros, sin embargo el hecho de no contar con un adecuado nivel de respuesta, es decir, que sea menor al 95\% considerado como un nivel adecuado o aceptable para dar resultados correctos. Los resultados a lo anterior muestran que será necesario establecer estrategias que permitan mejorar los resultados, ya que esto podrá garantizar que las personas podrán contar con mayores conocimientos, lo cual dará mayor fortaleza a la organización y le permitirá asegurar que el servicio prestado es de calidad y con ello dará tranquilidad a los inversionistas.

En segundo lugar se encuentra el tema de las generalidades de los costos con un nivel de 68\%, el cual incluye las bases y conceptos relacionados con la contabilidad (financiera, administrativa y de costos ); para ello además se debe conocer conceptos, clasificaciones y en general la teoría básica. Con los resultados anteriores se puede considerar que en este tema, que es básico para el conocimiento de los costos, tiene un nivel muy bajo, lo cual hace necesario establecer estrategias para que los alumnos y egresados mejoren sus bases, y con ello tener mejor desempeño de los temas posteriores del área de costos, ya que el conocer de manera correcta las bases de los costos, les permitirá no solo brindar un mejor servicio del trabajo que realizan, sino desarrollar y proponer procesos innovadores y legales que mejoren los resultados de la organización.

El tercer tema de acuerdo a las respuesta es el relacionado con los elementos del costo de producción, registro y reporte ya que tiene un nivel de $62 \%$ de respuesta correcta, lo cual indica que todo lo relacionado con la definición, descripción, clasificación e identificación, de cada uno de los elementos del costo, es decir, de materia prima, mano de obra y costos indirectos de fabricación, lo cual es de suma importancia para la generación del costo correcto de acuerdo al tipo de producto o servicio que se ofrece, ya que esto se convierte en la base del precio, y por lo tanto de la generación de la ganancia de la organización. Sin embargo, el resultado presentado es muy bajo, ya que muestra un nivel no aceptable considerando que un $95 \%$ de conocimiento es lo más adecuado. Por lo anterior es necesario que los responsables de los programas académicos realicen propuestas para mejorar los resultados, tanto académicamente como laboralmente, ya que ello garantiza que los alumnos y egresados realmente son competentes para desarrollar dicha actividad.

En cuarto y último lugar se encuentra el tema relacionado con los sistemas de costos de productos y servicios, tanto para órdenes de trabajo o para el sistema por procesos continuos, los cuales muestran un resultado del $59 \%$ de respuestas correctas, lo cual es poco aceptable ya que el tema considera que los sistemas de costos basados en diferentes formas de trabajo, así como los subsistemas derivados tales como la producción conjunta, los co-productos y los subproductos. Lo anterior permite que al contar con este nivel de conocimiento los responsables de los recursos incrementen su capacidad de creatividad e innovación, a un nivel mayor, sin estar fuera de la parte legal. La importancia de este tipo de actividades permitirá ampliar y mejorar el desempeño de los alumnos en este tema y poder llevarlos a un nivel de expertos.

La hipótesis planteada en la presente investigación fue la de identificar si no hay deferencias en cuanto a conocimiento entre los temas de costos, rechazando la hipótesis ya que el resultado muestra que se tiene diferencia significativa con respecto a los diferentes temas de costos. 
Es importante mencionar que en general los costos son importantes porque permiten analizar el resultado final de la organización, y con ello poder garantizar, de acuerdo al sector en el que se desenvuelve la empresa, desarrollar estrategias de reducción de costos o bien mejorar en general la estructura del costos conforme a sus propios procesos, asegurando con ello un proceso de mejora constante con la organización.

De acuerdo con los resultados, es importante que la institución revise los procesos de enseñanza aprendizaje, para asegurar que los contenidos de los programas son aprendidos y aplicados directamente por los alumnos, quienes posteriormente podrán emplearlos en las actividades de la organización, asegurando con ello que el conocimiento realmente se adquiere y se aplica, para con ello formar mejores profesionistas que realmente podrán aplicar lo aprendido en la actividades de la organización, y asegurar además que podrán realizar mejoras efectivas en el corto plazo en la organización.

Se sugiere para futuras investigaciones hacer un instrumento en escala de likert, que permita analizar de con estadística multivariante los resultados obtenidos y con ello hacer propuestas de mejora en base a otro tipo de resultado más robusto.

Finalmente se concluye que los resultados obtenidos en el presente trabajo de investigación no muestran que los alumnos y egresados tienen un nivel aceptable de conocimientos, ya que el resultado muestra un conocimiento que nos es suficiente para calificar a los alumnos como expertos, cabe mencionar que las personas seleccionadas fueron aquellos con un nivel alto de calificaciones. Por lo anterior es recomendable que se realice una revisión a profundidad para detectar que es lo que está fallando en el proceso y hacer mejorar lo antes posible, para asegurar que los estudiantes saldrán realmente como expertos, que es el nivel que realmente requieren los egresados, para que realicen su trabajo con un alto nivel de trabajo y con un reconocimiento tal que sean considerados al menos en el corto plazco, como profesionistas expertos.

\section{Referencias:}

Accenture (2012). La cadena de suministro para el canal tradicional. Pulso Estratégico, 3, 15-17 [consultado 16 Nov 2013]. Disponible en: http://www.accenture.com/SiteCollectionDocuments/P DF/Accenture-Pulso-Estrategico.pdf

Amat, O. y Soldevila, P. (1998). Contabilidad de Gestión y de Costes. 2da ed. España: Ediciones Gestión 2000.

Bjornenak, T. \& Mitchell, F. (1999). A study of the development of the Activity-Based Costing journal Literature 1987-1998 (University of Pittsburgh Working Paper).

Byrnes, J. (2010). Islands of profit in a sea of red ink. New York: Portfolio - Penguin. Calle, R. y Makon, M. (2012). Cost estimation in public sector entities: A methodological proposal. International Journal of Public Budget, 78, 1-19.

Cagwin, D. \& Bouwman, M.J. (2002). The association between Activity-Based Costing and improvement in financial performance. Management Accounting Research, 75(1), 1-39.

Cooper, R., and Kaplan, R. S. (1998). The promise - and peril - of integrated cost systems. Harvard Business Review, 76 (4), 109.

Cuevas, C. F., Chavez, G., Castillo, J.A., Caicedo, N.M. \& Solarte, W.F. (2004). Costeo ABC ¿Por qué y cómo implantarlo? Estudios Gerenciales,92, 47-103. Recuperado de http://dspace.icesi.edu.co/dspace/ bitstream/10906/337/l/cfcuevasgchavez-jcastilloncaicedowsolarte-costeo_abc.pdf
Vanessa Carolina Hernández Monsreal; Patricia Hernández García; Ma. Guadalupe de Socorro Laura Dávalos Verástegui 
Demeere, N., Stouthuysen, K. \& Roodhooft, F. (2009). Time-driven Activity-Based |Costing in an outpatient clinic environment: development, relevance and managerial impact. Health Policy, 92(2), 296-304.

Emblemsvag, J. (2007). Using activity-based costing and economic profit to grow the bottom-line. Business Strategy Series, 8(6), 418-425.

Freeman, B., Haasz, S., Lizzola, S. y Seiersen, N. (2000). Managing your cost to serve. Supply Chain Forum: An International Journal, 1(1), 18-28.

García Colín, J. (2008) Contabilidad de costos. México, D.F.: McGraw-Hill/Interamericana Editores, SA de CV

Guerreiro, R., Rodrigues, S. y Vazquez, E. (2008). Costto-serve measurement and customer profitability analysis. International Journal of Logistics Management, 19(3), 389-407.

Guiltinan, J., Paul, G. \& Madden,T. (2007). Gerencia de marketing: Estrategias y programas. México: McGrawHill.

Hansen y Mowen (1996). Administración de Costos. Contabilidad y Control. International Thomson Editores, S.A. México.

Hernández Sampieri, R., Fernández Collado, C., y Pilar Baptista Lucio, M. P. (2014), Metodología de la investigación. México, D.F.: McGrawHill/Interamericana Editores, SA de CV

Hicks, D. (1998). El sistema de costos basado en las actividades (ABC). México: Alfaomega.

Kaszubski, M.A \& Ebben, S. (2004). Using ActivityBased Costing to implement behavioural cost initiative successfully. Journal of Facilities Management, 5(2), 184-192.

Marteau, S. y Perego, L. (2001). Modelo de Costo Basado en Actividades aplicado a consultas por trazadores de enfermedades cardiovasculares. Salud Pública de México, 43(1), 32-40.
Mejía Argueta, C. y Higuita Salazar, C. (2015). Costo de servir como variable de decisión estratégica en el diseño de estrategias de atención a canales de mercados emergentes. Estudios Gerenciales, 134(31), 50-61.

Morillo, M. (2001). Rentabilidad Financiera y Reducción de Costos, Actualidad Contable Faces, 4(4), 35-48.

Nakagawa, M. (2002). Gestáo estratégica de custos: conceitos, sistemas e implementaçào JIT/TQC (7a ed.). Sâo Paulo: Atlas.

Pérez de León, O. (1999). Contabilidad de Costos. México: Instituto Mexicano de Contadores Públicos, A.C. Ediciones Limusa.

Ramírez Padilla, D. N. (2008). Contabilidad administrativa. México, D.F.: McGrawHill/Interamericana Editores, SA de CV

Rodríguez-Maeso, J.E. (2010). El actual estado del arte en los sistemas ABC/ABM: un análisis e interpretación de la literatura internacional. Revista Iberoamericana de Contabilidad e Gestión, 5(15), 1-20.

Taj, S. y Berro, L. (2006). Application of constrained management and lean manufacturing in developing best practices for productivity improvement in autoassembly plant. International Journal of Productivity and Performance Management, 55(3-4), 332-345.

Tsai, W-H. \& Lai, C-W. (2007). Outsourcing or capacity expansions: application of Activity-Based Costing model on joint products decisions. Computers \& Operations Research, 34(12), 3666-3681.

Hernández Sampieri, R., Fernández Collado, C., y Pilar Baptista Lucio, M. P. (2014), Metodología de la investigación. México, D.F.: McGrawHill/Interamericana Editores, SA de CV.
Vanessa Carolina Hernández Monsreal; Patricia Hernández García; Ma. Guadalupe de Socorro Laura Dávalos Verástegui 